\title{
Design and Analysis of Novel Fractal Linear and Planar Array Antennas for Satellite Applications
}

\author{
V. A. Sankar Ponnapalli ${ }^{1}$, P. V. Y. Jayasree ${ }^{2}$ \\ ${ }^{1,2}$ Department of Electronics and Communications Engineering, GITAM University, Visakhapatnam 530045, India \\ *corresponding author, E-mail: vadityasankar3@gmail.com
}

\begin{abstract}
This article proposes a new geometric design methodology for the systematic expansion of fractal linear and planar array antennas. Using this proposed geometric design methodology any deterministic polygon shape can be constructed. In this article, two element fractal linear and triangular array antennas are examined using proposed methodology up to four iterations of two expansion factors. Due to the repetitive nature of the proposed geometric design methodology, both linear and planar fractal arrays shows multi-beam behavior with excellent array factor properties. The behavior of the proposed arrays shows better performance than linear and planar fractal array antennas generated by concentric circular ring sub array geometric generator. Triangular planar fractal array of expansion factor two at fourth iteration achieved a single valued beam width of $3.8^{0}$ with -31.6 side lobe level. The suggested fractal arrays are analyzed and simulated by MATLAB-13 programming.
\end{abstract}

\section{Introduction}

Array antennas play a key role in military and defense applications due to their less side lobes, fast beam scanning, high directive gain and resolution properties [1]-[3]. Fractal array antennas are also find applications in both military and civilian due to their high gain with less number of antenna elements, UWB behavior, multiband characteristics and abated mutual coupling and side lobes [4]-[6]. Design methodologies of fractal geometric nature are plays a vital role in the designing of linear, planar and conformal fractal array antennas. Wide band and multiband characteristics of fractal array antennas depends on fractal geometric nature and how they are generated with different expansion factors. Hence these array antennas are geometry based not material based. But only a small number of design methodologies are available for the design of fractal array antennas [7], [8]. Concentric circular ring sub array geometric generator is one of the pioneer design methodologies for the generation of linear and planar fractal array antennas. Different types of fractal array antennas like triadic cantor linear and planar fractal arrays, Sierpinski carpet and triangular, square fractal arrays, pentagonal, hexagonal, heptagonal and octagonal fractal array antennas have implemented using this design methodology [9], [10].
Conformal fractal array antennas like Menger sponge and 3-D Sierpinski gasket array antennas are generated by concentric sphere 3-D array geometric generator and these arrays find applications in military aircrafts, ships, land vehicles and missiles because of their fractal and conformal natures [11]. To overcome the basic draw backs in previous design methodologies concentric elliptical ring sub array design methodology proposed for the generation of deterministic fractal antenna arrays and this methodology shows better performance in various array factor properties [12], [13].

Another type of fractal array antennas are also available, they are nature inspired fractal random array antennas [14], cantor fractal linear array for even number of antenna elements [15] and Haferman carpet antenna array [16]. To reach the constraints of satellite and space communication systems like high end of coverage directivity, less side lobe level hybrid-fractal direct radiating antenna arrays presented in [17]. Dragon, flap, twig fractal array antennas are proposed for celestial communication surveillance and wireless communication systems in [18]. The cantor ring array designed by polyadic design methodology for less side lobe levels is also the famous fractal array antennas [19]. To avoid overlaps and gaps between the elements like in conventional fractal arrays a new type of fractal arrays having fractal boundaries named as fractile arrays are proposed in [20]. New types of fractal elements based on Sierpinski fractal are proposed in [21], to reduce overall size and antenna elements of an array with Euclidean-based antenna elements. Due to their ease of generation and a variety of applications in space and other advanced communication systems, fractal arrays become more popular and wide amount of research done on fractal antennas but articles related to this type of work are relatively less.

This paper mainly concentrated on the design of linear and planar triangular fractal array antennas using concentric elliptical ring sub array generator geometric design methodology. The paper is organized as follows. Section 2 introduces the concentric elliptical ring sub array geometric design methodology and how it can generate various fractal arrays. Section 3 explains the expansion of both linear and planar fractal array antennas using the concentric elliptical 
ring sub array design methodology. Section 3 shows results of the proposed fractal array antennas. Finally, conclusion draws in section 5 of this article.

\section{Proposed Design Methodology: A Fractal Array Generator}

Novel geometric design methodologies will be helpful for the improvement of fractal array antenna concert. In this manner, this paper discusses a concentric elliptical sub array generator for the expansion of linear and planar fractal array antennas. This methodology replicates the concentric circular ring sub array generator but in this case circular generator is substituted with elliptical generator as shown in Figure. 1 and radius is the significant parameter in concentric circular sub array generator but eccentricity is the important consideration in this design methodology. Eccentricity can be varies using major axis (a) and minor axis (b). Here only two stages of growth are showing in Figure. 1 and generating array factor is expressed in (1) and resulting array factor expression is defined in (2), but this can be generated up to infinite iterations (P). The basic sub array generator can be repeated again and again according to number of iterations. The number of sub array antenna elements should be depending on the expansion factor (S).

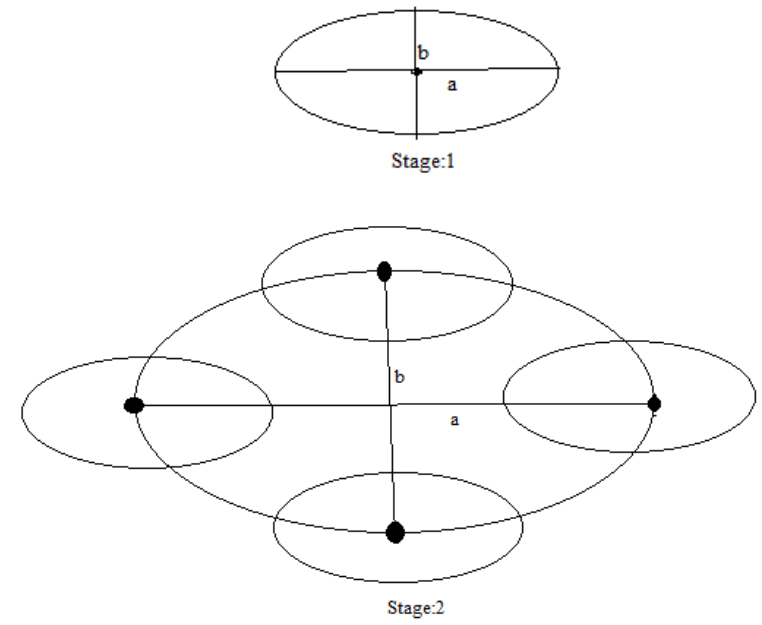

Figure 1. Concentric elliptical sub array generator for stage:1 and stage:2.

The consequent generating array factor for concentric elliptical ring array can be expressed as,

$$
\begin{aligned}
& G A(\theta, \phi)=\sum_{m=1}^{M} \sum_{n=1}^{N} I_{m n} e^{j k \Psi_{m n}} \\
& A . F_{P}(\theta, \phi)=\prod_{P=1}^{P}\left[\sum_{m=1}^{M} \sum_{n=1}^{N} I_{m n} e^{j k S^{P-1} \Psi_{m n}}\right]
\end{aligned}
$$

$\Psi_{m n}=\sin \theta\left(a \cos \phi_{n} \cos \phi+b \sin \phi_{n} \sin \phi\right)-\sin \theta_{0}\left(a \cos \phi_{n} \cos \phi_{0}+b \sin \phi_{n} \sin \phi_{0}\right)$

where $\mathrm{I}_{\mathrm{mn}}$ is the current excitations, $\mathrm{k}$ is the wave number, $\mathrm{S}$ is the expansion factor, $\mathrm{P}$ is the iteration number, $\theta_{0}$ and $\phi_{0}$ are the beam steering angles, $\phi_{\mathrm{n}}=(\mathrm{n}-1)(2 \pi / \mathrm{n})$, a is the major axis and $\mathrm{b}$ is the minor axis, $\mathrm{M}$ is the resultant concentric rings, $\mathrm{N}$ is the total number of elements.

\section{Design and Analysis of Two Element Linear Fractal Array Antenna}

Basic geometric generator of proposed array antenna starts with two antenna elements and it can be generated up to infinte iterations. This paper considers up to four iterations having same distance $(d=\lambda / 2)$ between the antenna elements and maintained for expansion factors (S) of both one and two. Nearly one third of the antenna elements can be thinned in each iterations due to recursive nature of the proposed methodology. Expansion factor of any fractal array chooses the number of antenna elements and their positions. In this article two types of expansion factors are considered for expansion of linear fractal array antenna. The array factor of the proposed array can be changes depending on the expansion factor (S). Figure. 2 illustrate the proposed array for an expansion factor of one. Nearly $20-30 \%$ antenna elements thinned in second, third and fourth iterations due to recursive nature of the methodology. The array factor of linear fractal array antenna is shown below,

$$
A \cdot F_{P}(\theta, \phi)=\prod_{P=1}^{4}\left[\sum_{m=1}^{1} \sum_{n=1}^{2} I_{m n} e^{j k^{P-1} \Psi_{m n}}\right]
$$

where $I_{m n}$ is the current amplitudes, $k$ is the wave number, $\mathrm{S}$ is the expansion factor, here $\mathrm{S}=1, \mathrm{P}$ is the iteration number, $\mathrm{m}$ is the concentric rings, $\mathrm{n}$ is the number of elements.

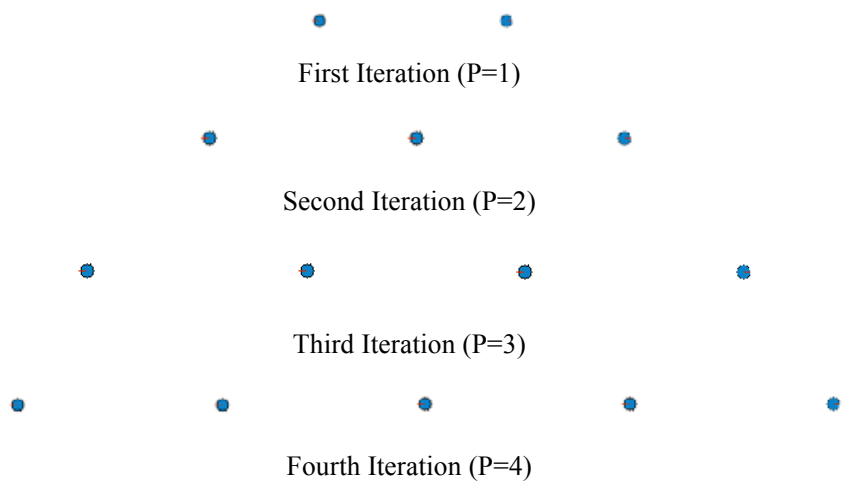

Figure 2. First four iterations of linear fractal array antenna for an expansion factor of one 
Array factor of the linear fractal array antenna for an expansion factor of two is illustrated in (5). Actually difference between this array and above array is expansion factor. Depending on the expansion factor behavior of the fractal array can be changes. Geometrical construction of two-element linear fractal array for an expansion factor of two is shown in Figure. 3. Due to its expansion nature no elements thinned in this array configuration.

$$
\begin{aligned}
& A . F_{P}(\theta, \phi)=\prod_{P=1}^{P}\left[\sum_{m=1}^{M} \sum_{n=1}^{2} I_{m n} e^{j k 2^{P-1} \Psi_{m n}}\right] \\
& \text { First Iteration }(\mathrm{P}=1) \\
& \text { Second Iteration }(\mathrm{P}=2) \\
& \text { Third Iteration }(\mathrm{P}=3)
\end{aligned}
$$

Figure 3. First three iterations of two element linear fractal array antenna for an expansion factor of two

\section{Design and Analysis of Triangular Planar Fractal Array Antenna}

Basic triangular array starts with three elements and expanded up to four iterations in this paper. Same distance $(d=\lambda / 2)$ between the antenna elements maintained for both expansion factors (S) of one and two. Nearly one third of the antenna elements can be thinned in first iterations due to recursive nature of the proposed methodology. Genertion of this array is more simple and rapid beam forming algorithms will be helpful for easy generation fractal arrays up to infinite iteration. Expansion factor of any fractal array chooses the number of antenna elements and their positions. In this article two types of expansion factors are considered for the expansion of triangular planar fractal array antenna. The array factor of the proposed array can be changed depending on the expansion factor (S). Figure. 4 exemplify the proposed array for an expansion factor of one. Nearly 20-30\% antenna elements thinned in second, third and fourth iterations due to recursive nature of the methodology. The array factor of triangular planar fractal array antenna is shown below,

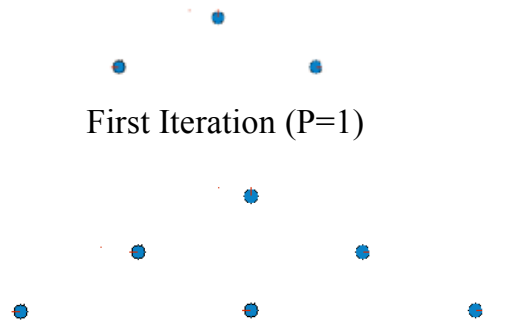

Second Iteration $(\mathrm{P}=2)$

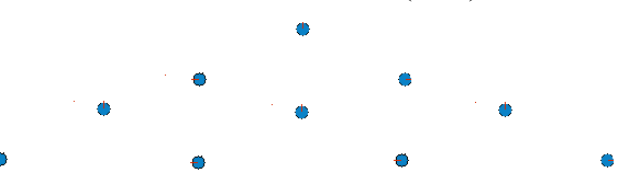

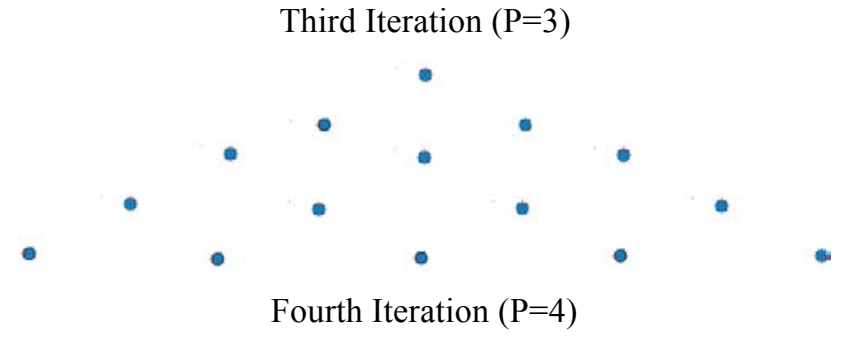

Figure 4. First four iterations of triangular planar fractal array antenna for an expansion factor of one.

$$
A . F_{P}(\theta, \phi)=\prod_{P=1}^{4}\left[\sum_{m=1}^{1} \sum_{n=1}^{3} I_{m n} e^{j k^{P-1} \Psi_{m n}}\right]
$$

where $I_{m n}$ is the current amplitudes of triangular array antenna, $\mathrm{k}$ is the wave number, $\mathrm{S}$ is the expansion factor, here $\mathrm{S}=1, \mathrm{P}$ is the iteration number, $\mathrm{m}$ is the concentric rings, $\mathrm{n}$ is the total number of elements of this array.

Array factor of the triangular planar fractal array antenna for an expansion factor of two is illustrated in (7). Actually difference between this array and above array is expansion factor. Depending on the expansion factor behavior of the fractal array can be changes. Geometrical construction of this fractal array for an expansion factor of two is shown in Figure. 5. Due to its expansion nature no elements thinned in this array configuration.

$$
A . F_{P}(\theta, \phi)=\prod_{P=1}^{P}\left[\sum_{m=1}^{M} \sum_{n=1}^{3} I_{m n} e^{j k 2^{P-1} \Psi_{m n}}\right]
$$

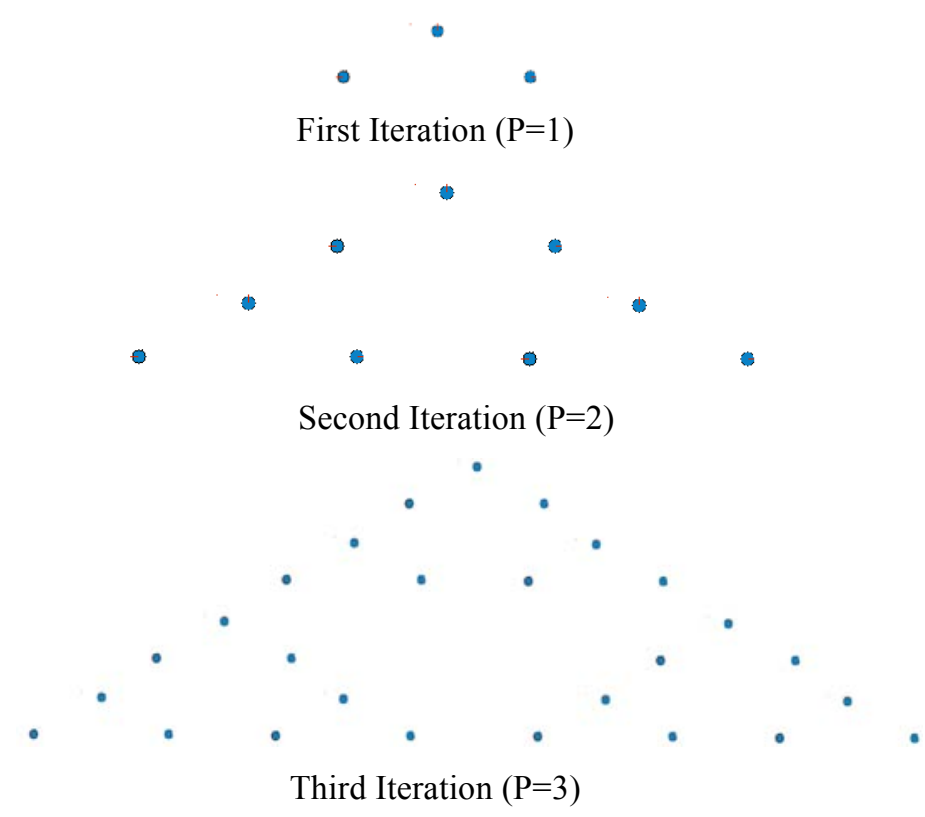

Figure 5. First three iterations of triangular planar fractal array antenna for an expansion factor of two

\section{Discussion on Results}

This paper focuses on the design of linear and planar fractal antenna arrays using concentric elliptical sub array 
geometric design methodology. Basic arrays starts with two and three elements and it can extend up to infinite iterations but this paper examined up to four iterations for expansion factors of one and two. While increasing the iterations of both fractal arrays there is a simultaneous decrease observed in resolution of both expansion factors. Proposed linear and triangular planar array antennas are shows better performance in all array factor properties than concentric circular geometric methodology.

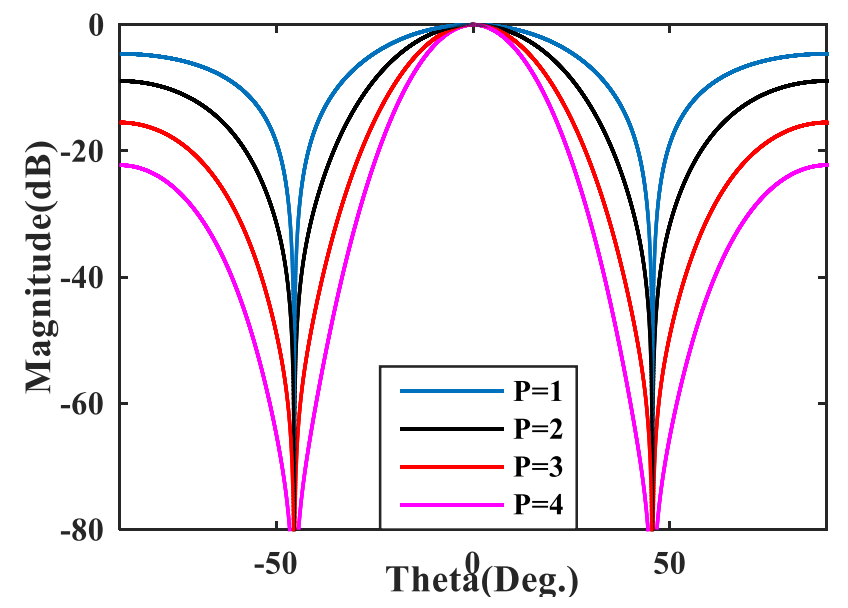

(a)

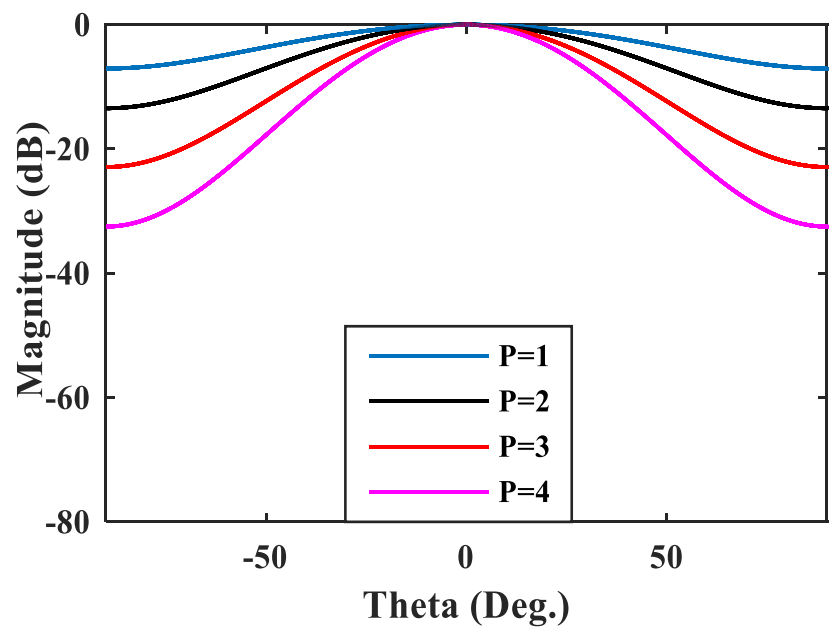

(b)

Figure 4. Array factors of linear fractal array antennas generated by concentric (a) elliptical and (b) circular ring sub array generators for $S=1$ up to four iterations.

An abated HPBW has observed in both cases of linear and triangular planar fractal array antenna than fractal array antennas generated by concentric circular ring array generator as shown in Fig.6(a) and 9(b). In the first case no side lobes observed like in linear fractal array antenna generated by circular generator for an expansion factor of one, but nearly half of the HPBW reduced in all iterations than the linear fractal array generated by circular generator. At the fourth iteration of both arrays for expansion factor two the HPBW is very narrower less side lobe levels. This indicates the proposed array antennas will be useful for the long distance and the satellite communication systems.

The distance between the main lobe and adjacent side lobes in degrees is generally known as side lobe level angle. Wider side lobe level angle with less side lobes improves the signal to noise ratio and signal to inter symbol interference. Another enhancement of elliptical sub array generator is orientation; this means shape of the ellipse is based on major and minor axis depending on the space constrains these axis's can be interchanged. This is not possible in circular geometric generator because eccentricity of the circle is zero. Fig. 6(b) and 9(b) exemplifies antenna elements increases with number of iteration.

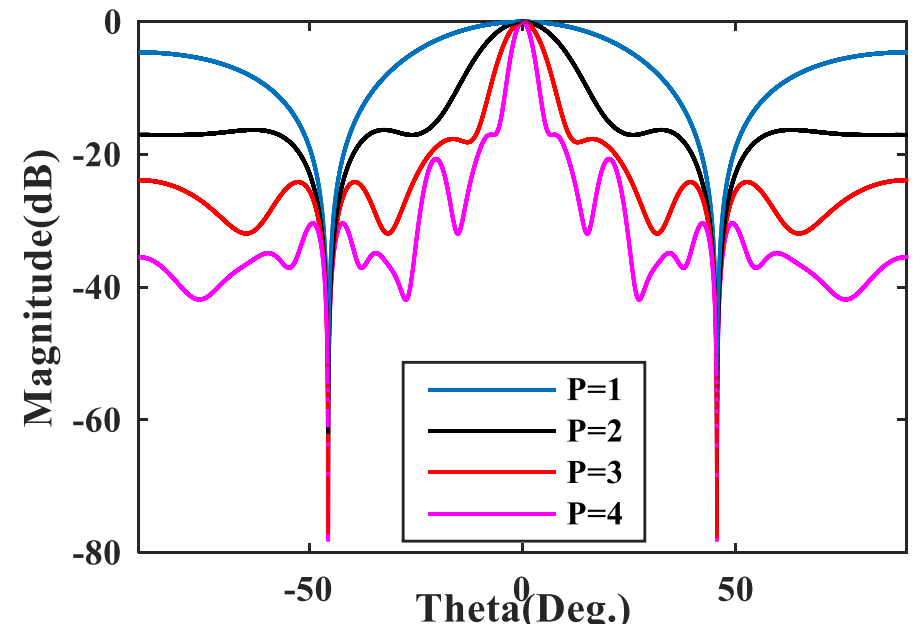

(a)

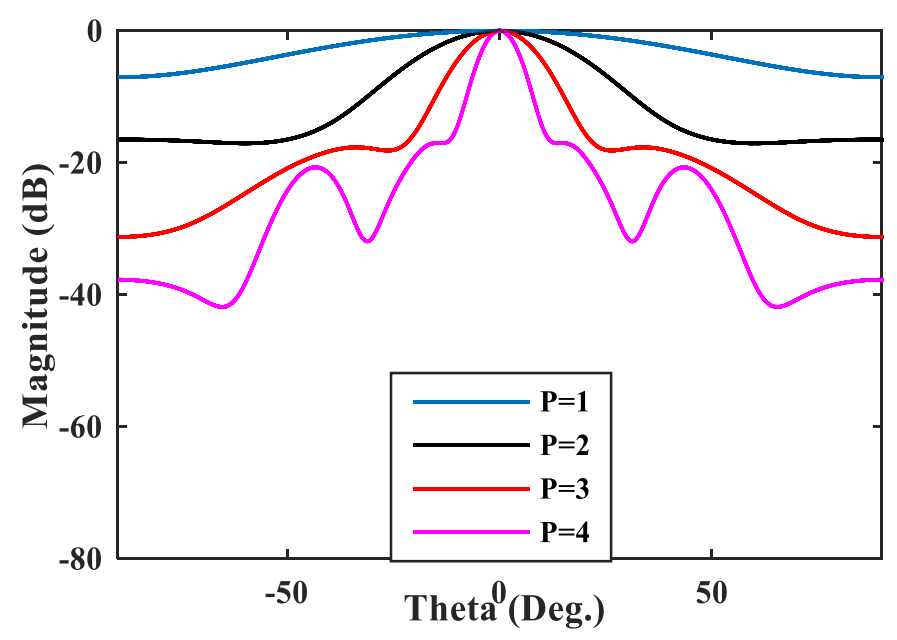

(b)

Figure 5. Array factors of linear fractal array antennas generated by concentric (a) elliptical and (b) circular ring sub array generators for $\mathrm{S}=2$ up to four iterations. 


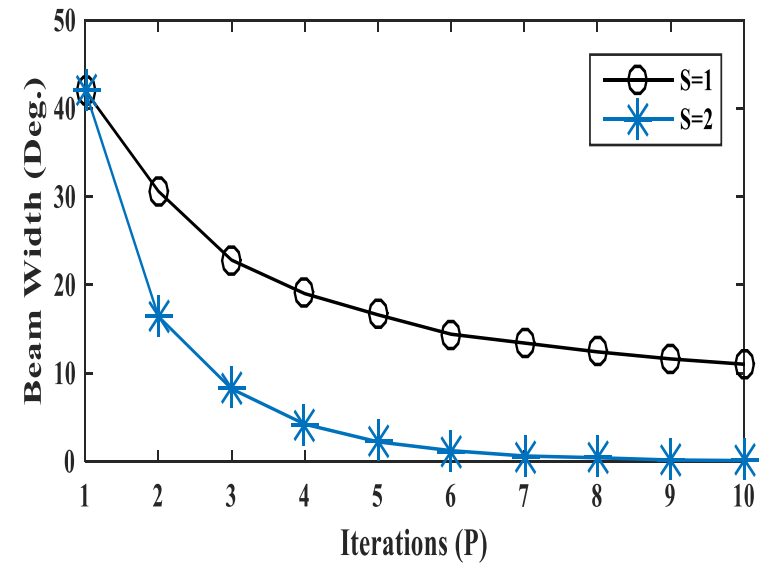

(a)

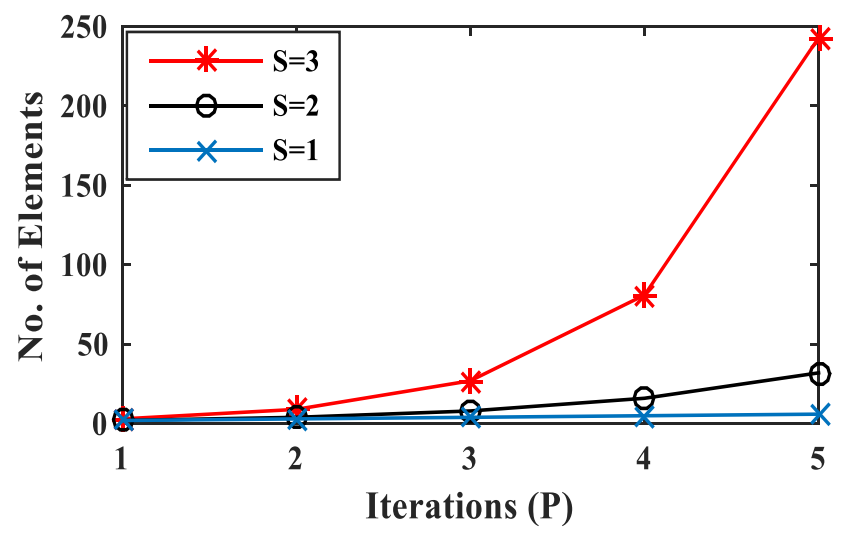

(b)

Figure.6. (a) Number of iterations with corresponding beam widths and (b) Number of iterations with number of elements.

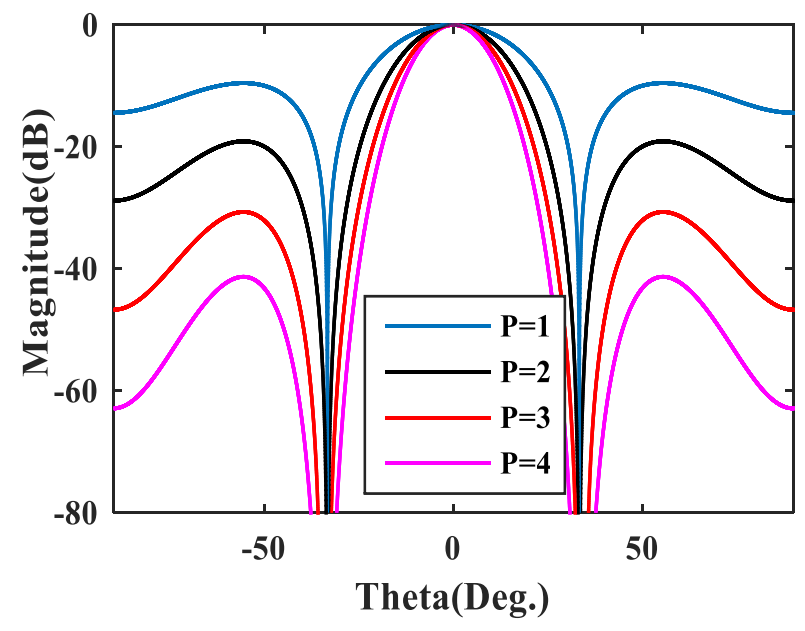

(a)

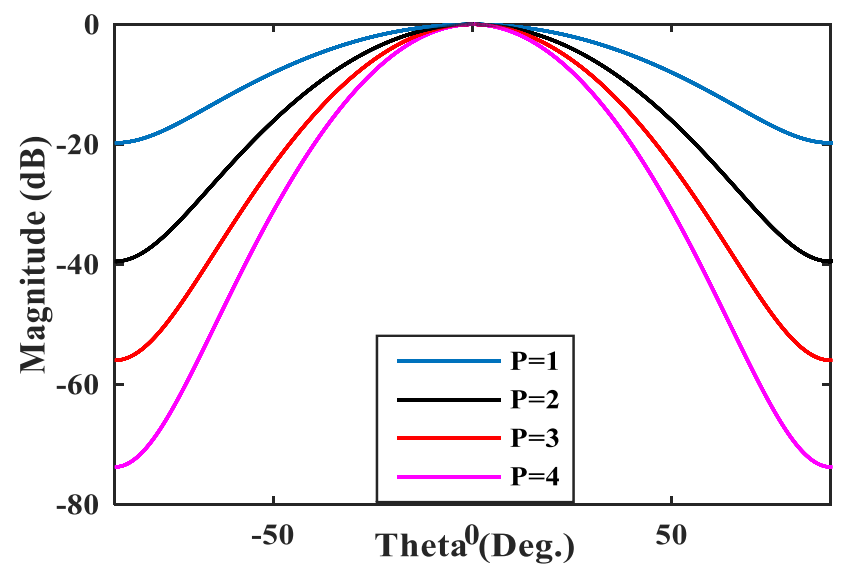

(b)

Figure 7. Array factors of triangular fractal planar array antennas generated by concentric (a) elliptical and (b) circular ring sub array generators for $S=1$ up to four iterations.

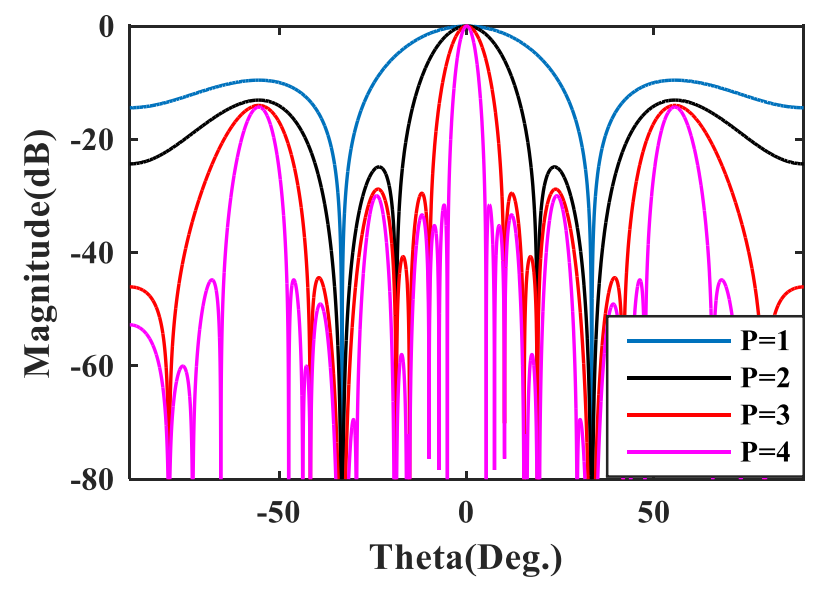

(a)

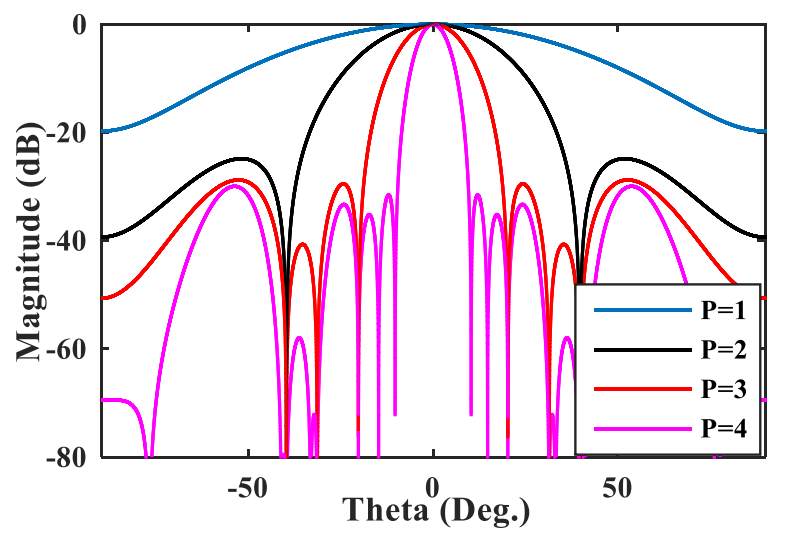

(b)

Figure 8. Array factors of triangular fractal planar array antennas generated by concentric (a) elliptical and (b) circular ring sub array generators for $S=2$ up to four iterations. 


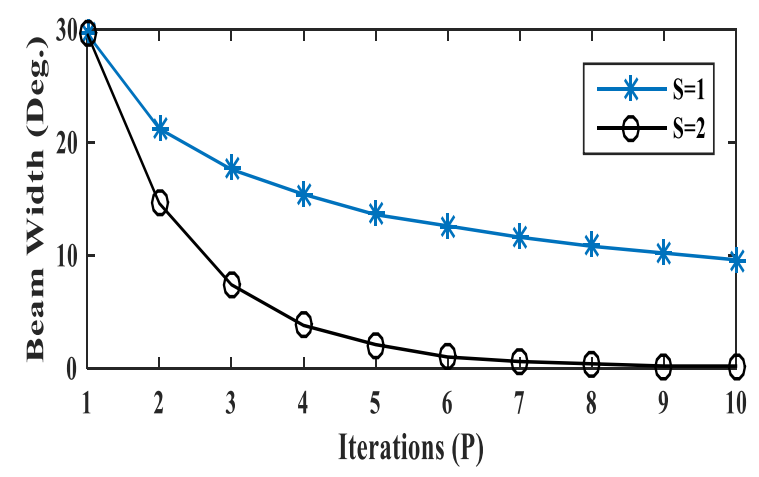

(a)

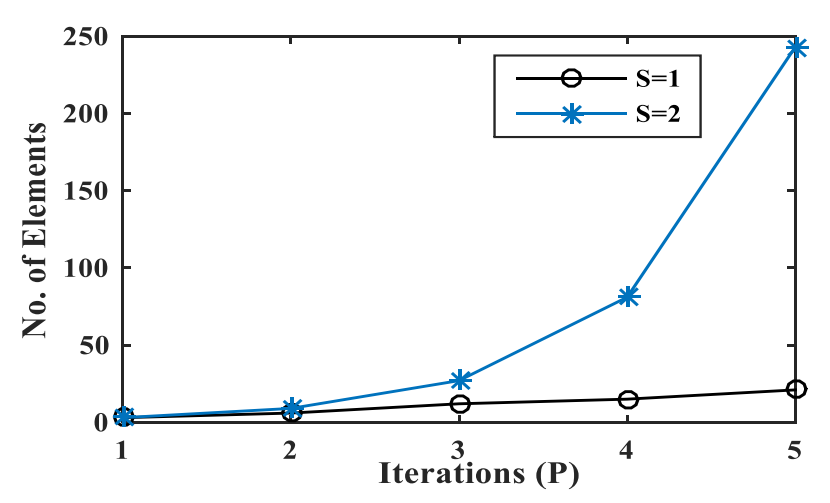

(b)

Figure.9. (a) Number of iterations with corresponding beam widths and (b) Number of iterations with number of elements.

Table .I. Comparison of SLL, HPBW, SLL Angle of both linear fractal arrays generated with concentric elliptical and circular sub array generators for expansion factors $S=1$ and 2 up to four iterations.

\begin{tabular}{|c|c|c|c|c|c|c|c|c|c|c|}
\hline $\begin{array}{c}\text { Iteratio } \\
\text { ns } \\
(\mathrm{P})\end{array}$ & \multicolumn{9}{|c|}{$\begin{array}{c}\text { Two-Element Linear Fractal Array Antenna } \\
\text { (Concentric elliptical sub array geometric generator) }\end{array}$} & \multicolumn{3}{c|}{$\begin{array}{c}\text { Two-Element Linear Fractal Array Antenna } \\
\text { (Concentric circular ring sub array geometric } \\
\text { generator) }\end{array}$} \\
\hline \\
\hline
\end{tabular}

Table .II. Comparison of SLL, HPBW, SLL Angle of both linear fractal arrays generated with concentric elliptical and circular sub array generators for expansion factors $S=1$ and 2 up to four iterations.

\begin{tabular}{|c|c|c|c|c|c|c|c|c|c|c|c|c|}
\hline \multirow{3}{*}{$\begin{array}{c}\text { Iteratio } \\
\mathrm{ns} \\
(\mathrm{P}) \\
\mathrm{S}\end{array}$} & \multicolumn{6}{|c|}{$\begin{array}{l}\text { Triangular Fractal Planar Array Antennas } \\
\text { (Concentric elliptical sub array geometric generator) }\end{array}$} & \multicolumn{6}{|c|}{$\begin{array}{c}\text { Triangular Fractal Planar Array Antennas } \\
\text { (Concentric circular ring sub array geometric } \\
\text { generator) }\end{array}$} \\
\hline & \multicolumn{3}{|c|}{ Expansion Factor $(\mathrm{S})=1$} & \multicolumn{3}{|c|}{ Expansion Factor $(\mathrm{S})=2$} & \multicolumn{3}{|c|}{ Expansion Factor $(\mathrm{S})=1$} & \multicolumn{3}{|c|}{ Expansion Factor $(\mathrm{S})=2$} \\
\hline & $\begin{array}{l}\text { HPBW } \\
\text { (Deg.) }\end{array}$ & $\begin{array}{l}\text { Side } \\
\text { lobe } \\
\text { level } \\
(\mathrm{dB})\end{array}$ & $\begin{array}{l}\text { SLL } \\
\text { Angle } \\
\text { (Deg.) }\end{array}$ & $\begin{array}{l}\text { HPBW } \\
\text { (Deg.) }\end{array}$ & $\begin{array}{l}\text { Side } \\
\text { lobe } \\
\text { level } \\
(\mathrm{dB})\end{array}$ & $\begin{array}{l}\text { SLL } \\
\text { Angle } \\
\text { (Deg.) }\end{array}$ & $\begin{array}{l}\text { HPBW } \\
\text { (Deg.) }\end{array}$ & $\begin{array}{l}\text { Side } \\
\text { lobe } \\
\text { level } \\
(\mathrm{dB})\end{array}$ & $\begin{array}{l}\text { SLL } \\
\text { Angle } \\
\text { (Deg.) }\end{array}$ & $\begin{array}{r}\text { HPBW } \\
\text { (Deg.) }\end{array}$ & $\begin{array}{l}\text { Side } \\
\text { lobe } \\
\text { level } \\
(\mathrm{dB})\end{array}$ & $\begin{array}{l}\text { SLL } \\
\text { Angle } \\
\text { (Deg. } \\
\text { ) }\end{array}$ \\
\hline 1 & 29.6 & -9.5 & 53.1 & 29.6 & -9.5 & 53.1 & 60.8 & $-\infty$ & - & 60.8 & $-\infty$ & - \\
\hline 2 & 21.2 & -19.0 & 55.4 & 14.6 & -24.9 & 23.5 & 42.8 & $-\infty$ & - & 29.0 & -24.8 & 52.5 \\
\hline 3 & 17.6 & -30.7 & 54.1 & 7.4 & -29.5 & 11.5 & 35.4 & $-\infty$ & - & 14.8 & -29.7 & 25.4 \\
\hline 4 & 15.4 & -41.3 & 55.8 & 3.8 & -31.6 & 6.2 & 30.6 & $-\infty$ & - & 7.4 & -31.6 & 12.8 \\
\hline
\end{tabular}

\section{Conclusions}

Linear and planar fractal arrays generated by concentric elliptical ring sub array generator are new type of fractal antenna arrays. These arrays showed better performance in various array factor properties than the previous design methodologies. This paper investigated these two fractal array antennas with different eccentric values and for expansion factors of 
one and two. This methodology shows better performance in orientation of the shape, Side lobe level, beam width and SLL angle rather than concentric circular ring sub array geometric generator. HPBW achieved a single digit value at the fourth iteration of both arrays for an expansion factor two. This design methodology and proposed arrays can be helpful for the generation of multiple-beams with different array factor properties using a single aperture fractal array antenna.

\section{References}

[1] C.A.Balanis, Antenna Theory: Analysis and Design, 2nd ed., New York, USA: John Wiley \& Sons, 1997.

[2] Robert J. Mailloux, Phased Array Antenna Handbook, 2nd ed., Massachusetts, USA: Artech House, 2005.

[3] R. C. Hansen, Phased Array Antennas, 2nd ed., New Jersey, USA: John Wiley \& Sons, 2009.

[4] John. D. Kraus and Ronald .J. Marhefka, Antennas: For All Application, 3rd ed., New York, USA: McGrawHill, 1997.

[5] Douglas H. Werner and Suman Ganguly, An overview of fractal antenna engineering research, IEEE Trans. Antennas Propag. Mag., 45 (2003), 38-57. doi: 10.1109/MAP.2003.1189650

[6] Steven. R. Best, Small and Fractal Antennas, in Modern Antenna Handbook, New York, USA: John Wiley \& Sons, 2008.

[7] D.H.Werner and R.Mittra, Frontiers in Electromagnetics, USA:IEEE Press, 2000.

[8] C. Puente Baliarda and R. Pous, Fractal design of multiband and low side-lobe arrays, IEEE Trans. Antennas and Propag. MAG., 44 (1996), 730-739. doi: 10.1109/8.496259

[9] Douglas H. Werner et al, Fractal antenna engineering: the theory and design of antenna arrays, IEEE Trans. Antennas Propag. Mag.,41(1999),37-59. doi: $10.1109 / 74.801513$

[10] V.A.Sankar Ponnapalli and P.V.Y.Jayasree, Heptagonal fractal antenna array for wireless communications, Microelectronics, Electromagnetics and Telecommunications -LNEE Series, 372 (2016), 387394. doi: 10.1007/978-81-322-2728-1_34

[11] Waroth Kuhirun, A new design methodology for modular broadband arrays based on fractal tilings, Ph.D. Thesis, Department of Electrical Engineering, Pennsylvania State University, USA, 2003.

[12] V.A.Sankar Ponnapalli and P.V.Y.Jayasree, Novel design methodology for the generation of deterministic fractal antenna arrays, IEEE International Microwave and RF Conference (IMaRC), 2015, 337-339. doi: 10.1109/IMaRC.2015.7411395

[13] V. A. Sankar Ponnapalli and P. V. Y. Jayasree, Design of multi-beam rhombus fractal array antenna using new geometric design methodology, Progress In Electromagnetics Research C, 64(2016), 151-158. doi: 10.2528/PIERC16031202

[14] Y. Kim and D.L. Jaggard, The fractal random array, IEEE Trans. Antennas Propag. Mag., 74 (1986), 1278 1280. doi: 10.1109/PROC.1986.13617

[15] V. Srinivasa Rao and V.A. Sankar Ponnapalli, Study and analysis of fractal linear antenna arrays, IOSRJECE, 5 (2013), 23-27. doi: 10.9790/2834-0522327

[16] Rowdra Ghatak, Anirban Karmakar and Dipak R. Poddar, Evolutionary optimization of haferman carpet fractal patterned antenna array, International J. of RF and Microwave Computer-Aided Engineering, 25 (2015), 719-729. doi: 10.1002/mmce.20911

[17] Katherine Siakavara, Hybrid fractal direct radiating antenna arrays with small number of elements for satellite communications, IEEE Trans. Antennas Propag.Mag.,58(2010),2102-2106. doi: 10.1109/TAP.2010.2046868

[18] Ahmed Najah Jabbar, New Elements concentrated planar fractal antenna arrays for celestial surveillance and wireless communications, ETRI J., 33 (2011), 849856. doi: 10.4218/etrij.11.0111.0036

[19] D. L. Jaggard and A. D. Jaggard, Cantor ring arrays, IEEE International Symposium on Antennas and Propagation Digest, 2(1998), 866-869. doi: 10.1109/APS.1998.702076

[20]D. H. Werner, Waroth.Kuhirun and P.L. Werner, Fractile arrays: A new class of tiled arrays with fractal boundaries, IEEE Trans. Antennas Propag. Mag., 52 (2004), 2008-2018. doi: 10.1109/TAP.2004.832327

[21] J. Anguera, G. Montesinos, C. Puente, C. Borja, and J. Soler, "An Under-Sampled High Directivity Microstrip Patch Array with a Reduced Number of Radiating elements Inspired on the Sierpinski Fractal", Microwave and Optical Technology Letters, 37 (2003), 100-103. doi: 10.1002/mop.10836 